\title{
Planning the Development of Electricity Grids in Developing Countries: An Initial Approach Using Agent Based Models
}

\author{
Jose F. Alfaro, Shelie A. Miller
}

\begin{abstract}
This paper presents a proof of concept model for the electrification process of developing countries. Considering the general needs and characteristics in those countries is important as they share factors far from the developed world situation. However, enough flexibility is included to allow implementation in individual countries with unique circumstances.

To achieve a robust yet flexible model, Agent Based Modeling is utilized. The model is built in the NetLogo architecture, which combines user interphases and geographic information extensions for adaptability.

The result is a tool that addresses a gap in the literature. Although models for developing countries exist, they fail to adequately capture the characteristics of their energy sectors. Agent Based Modeling allows the modeling of the electrification process through dynamic agents that do not focus on historical data or the normal development path followed by developed countries.

The model's strength is in the ease of scenario building so that policy makers and researchers can see the impact of decisions on the overall process. This also allows the stakeholders to quickly and easily seek the paths desired based on their objectives and identify the catalysts needed for those results.
\end{abstract}

Index Terms-ABM, Developing Countries, Electricity Grids, Modeling.

\section{INTRODUCTION}

$\mathrm{D}$ EVELOPING countries face the challenge of providing higher living standards for their population, protecting their natural resources, and keeping check on their greenhouse gas emissions. Electrification in bottom of the pyramid nations is a step that can highly affect other development factors for their population. The availability of electricity frees manpower for other income generating activities, reduces time spent gathering resources and performing domestic tasks, makes health resources available bettering communities' overall health and lengthens the time that students can spend on their education through artificial

This work was supported by the National Science Foundation.

J. F. Alfaro is a NSF Graduate Student Fellow with the Center for Sustainable Systems at the University of Michigan, School of Natural Resources and Environment, Ann Arbor, MI 48103 (phone: 734-764-1412; email: jfalfaro@umich.edu).

S. A. Miller is with the Center for Sustainable Systems at the University of Michigan, School of Natural Resources and Environment, Ann Arbor, MI 48103 (email: sheliem@umich.edu). light (International Energy Agency, 2010). Sources of jobs and an increased security are also achieved by electrification (Reiche, Covarrubias, \& Martinot, 2000).

There are favorable as well as unfavorable paths that can be followed towards electrification from environmental, economic and social points of view. Providing a robust tool that helps those countries plan their electrification allows them to make interventions at the beginning of the process giving them the highest probability for effective change.

\section{A. Objective}

Our objective is to develop a model that will provide an efficient tool for planning of the electric grid in developing nations through scenario building. This model is to capture the general characteristics of countries at the bottom of the pyramid. Further the model is to have flexibility so that it can be implemented in different countries to take into account their unique circumstances. This paper presents a proof-ofconcept model to lay the groundwork for a more robust model that incorporates more realistic scenarios.

To achieve these objectives we are using Agent Based Modeling (ABM) in the architecture of NetLogo (Wilensky, 1999). ABM allows researchers to model complex systems by representation of the actors (agents) in that system, the environment where they interact and the rules they utilize for their interaction and decision making. This is a modeling technique that permits the coding of a general model with high flexibility and adaptability for unique situations.

\section{B. A Gap in the Literature}

Although energy and electricity modeling is common, most literature revolves around the developed world. In the cases where models have been created or applied in the developing nations the unique characteristics of these countries are not properly captured (Urban, Benders, \& Moll, 2007). These models focus on econometric and engineering approaches that fail to capture the presence non-economic trading schemes and the leap-frog effect where countries adopt technologies faster than historical rates (Bhattacharyya \& Timilsina, 2010). ABM allows the modeler the flexibility to incorporate these and other issues unique to the countries analyzed by incorporating micro-interactions.

Developing nations, of course, are not homogeneous and those at the bottom of the pyramid, with little infrastructure, stand to 
reap the largest benefit from these planning efforts. It is precisely those countries that lag furthest behind in the Millennium Development Goals (MDG) and although there is no MDG specific to electricity, the achievement of those goals depends highly on the availability of clean cooking facilities and power (International Energy Agency, 2010). It is this gap in the literature that we hope to address.

\section{Energy Sector in Developing Countries}

In general, developing countries' energy sectors share characteristics that make them unique. Developing countries have a high reliance on traditional biomass. These sources of energy are not only detrimental to human health and the environment but also add complexity to the modeling demands since they are obtained in informal ways that are not captured by econometrics. Beyond the use of traditional sources of energy, bottom of the pyramid countries rely heavily on the use of fossil fuels for their electricity. Further there is a large urban-rural divide with respect to population density, income, access to modern energy and existence of infrastructure. These countries are also characterized by slow technology diffusion (Bhattacharyya \& Timilsina, 2010). Finally they share particular government structures and energy sector management. The general structure is for a central government that sets prices for energy and makes decisions on infrastructure with an agency devoted to attend to rural development and normally a private or government owned company that is charged with the grid development in urban areas where electrification may be profitable.

The demographics of developing countries are critical to the energy sector and the planning of its infrastructure. The urban-rural gap includes a difference in density where urban populations are highly compact and rural populations are dispersed through the country in small low-density pockets. This divide also includes a large economic difference. People in rural areas tend to earn much lower incomes and tend to have informal trading available to them such as bartering or harvesting of natural resources (Urban et al., 2007). Urban populations tend to have a much better infrastructure that allows people connection to those networks, in our case the electric grid. Expanding that grid allows the government to service the growing urban populations at a cheaper price than having to establish a separate grid to a similar number of people. Rural populations however, are usually far from these networks and expanding the grid to their geographical areas may be cost prohibitive or impractical. That creates a positive scenario for rural areas to be serviced by separate micro-grids that can be supplied by renewable energy sources (Zerriffi \& Wilson, 2010).

\section{MODEL}

Creating an $\mathrm{ABM}$ is very similar to writing a story. Stories are characterized by four components: characters, setting, mood and plot. Having the four components of a story allows the writer and sometimes the reader to get a general impression of where the story will go. Analogously, ABM does not rely on an overall theory of the mechanisms it is trying to describe. Instead, $\mathrm{ABMs}$ are built by creating the small units that interact in a model (characters), providing them with a world to interact in (setting), determining the rules that govern the world (mood), and finally providing those characters with rules for their interaction both with each other and the world they are in (plot).

Once this has been done the model is allowed to interact providing the phenomenon of emergence. Emergence is a macro-level complex behavior that rises from micro-level simple interactions (Garcia, 2005).

\section{A. Agents (Characters)}

There are several kinds of agents in our model who interact with each other to create the mechanisms we seek. We have the following agent-types or breeds in our model: Government, Rural-Centers, Urban-Centers and Organizations. Each of those breeds owns separate parameters that they utilize for making decisions. Their parameters are different from agent to agent, so not all Urban-Centers will have the same value for a particular parameter or variable.

Government agents are in charge of making the final decision on awarding money and which energy projects get funding. The Government decides which proposal is going to be funded during each time period of the model. The Government breed contains only one agent.

Rural-Centers is a breed of agents that contains all of the rural populations. In our general model this breed is created by providing the model with the population in rural areas and the number of rural centers present in the desired country. The model then creates those centers at random locations in our world and assigns them a population number based on a normal distribution of the total rural population of the world. These agents also own a resource for energy. For our proof of concept we simplify the model by determining at random access to a renewable energy source: photovoltaic, small wind or micro hydro. In a stronger model, these agents may have access to more than one resource and one resource may supply more than one agent. The type of energy source fixes the cost of electrical projects in that population center to the costs of the renewable source.

Rural-Centers also own an electricity demand. This demand is determined utilizing the value from the IEA of $250 \mathrm{kWh} /$ year for each household and the typical size of a household in developing countries. For our model we are choosing a size of five people per household. The government is expected to cover the initial cost of the project establishment.

For our general model the world is made up of homogenous patches. Each patch can hold a population center or a government agency. The patch sizes and distances are length unit dimensions. Later utilizing a GIS built in extension of the software a modeler can assign actual coordinates in the desired cartographic projection system making the world a true representation of a desired country.

Urban-Centers are analogous to the Rural-Center agents. Urban-Centers contain the urban population in the world also distributed normally among the Urban-Center agents. Their demand, similar to Rural-Centers, is determined by IEA numbers that place it at $500 \mathrm{kWh} /$ year for each household (International Energy Agency, 2010). The biggest difference between Rural-Centers and Urban-Centers is the UrbanCenters access to the established grids. For simplification, Urban-Centers do not own a renewable resource but instead 
they have access to the fossil fuel based grids in the centers already. Later, the model can be strengthened by giving these agents access to larger renewable resources like off-shore wind and traditional hydro. Their electrification costs are the costs of expanding the grids plus the fixed and variable costs of such expansion.

Our final breed of agents, Organizations, contains agents that are in charge of identifying proposals for electrification of a segment of the population and applying for funding from the Government. When funding is supplied the Organizations build the projects and restart their proposal process. There are two agents in this breed, one in charge of the rural populations and one in charge of the urban populations. This follows general observations in the developing world. These two agents compete against each other as the Government is constrained to the amount of proposals it can fund each time period in the model.

\section{B. Space (Setting)}

Space in our model refers to the world that the agents will interact in. ABM in general, and NetLogo in particular, allow for flexibility in the development of the world and alternatively for inclusion of very specific data. In our efforts we model a space that will be general enough for initial assessment of developing countries but at the same time allow for the inclusion of GIS specific data for individual countries in later efforts.

Further, our space holds the global variables of the model. These variables are either hard coded in the model or are user inputs through the graphical user interface (GUI). Global Variables are accessible by any agent or patch at any time during the model but the value of the variable does not change between agents. The variable may change over the time series of the model, in which case all agents will see the change and have to use the new value of the variable moving forward.

\section{Context (Mood)}

The context of our model follows the mood of the stories in the developing countries. Countries, or in our model Government, are trying to provide electricity access to the general population while working with limited capital and trying to protect their natural resources and limit their greenhouse gas emissions. Following this mood the government could use any sort of decision parameters to award the proposal funding. Environmental concerns and population services may be used at different weights to change the behavior of the agents.

In this context our model goes through a time series where the Organizations try to supply electricity to the population centers by proposing projects to the Government. The Government makes a decision on which proposal is the best and funds that proposal. Organizations then use those funds to build the project desired.

\section{Decision Making (Plot)}

The decision-making and communication between agents is a critical part of the model. Decision-making is complicated and hard to represent. However, in our model decisionmaking does not have to be perfect. This is where the flexibility of the model becomes a major strength. This model does not attempt to predict the future but to paint the possible scenarios (outcomes of the story) that will come with the different decision making processes. With this in mind, decision-making becomes a major variable that modelers and stakeholders can use to implement this model as a planning and policy investigation tool.

In our general model there are two decision rounds that take place in each time step. First the Organizations make a decision on which proposal they will bring forth to the Government. To do this, the Organizations have two different techniques. The Rural-Organization (in charge of providing electricity to Rural-Centers) assigns a random grade to each Rural-Center. The grades are a floating random number from 0 to 100 assigned by a random number generator. The RuralOrganization then takes the agent with the highest grade as the agent that will be proposed for electrification and calculates the cost of the proposal based on the renewable resource available to that agent and the costs associated with that renewable resource. The total cost of the proposal is calculated as the capital cost needed for satisfying the population electricity demand plus the net present value of the annual cost of maintenance, operation and fuel for the energy source over a project time life of 20 years. This stochastic method tries to reflect the fact that some population centers have patronage, political influence and other factors that affect decisions. As a proof of concept model this is sufficient. As the model is strengthened, those factors can be calculated into the priority of the population centers.

The Urban-Organization has to compete for the funding by providing a proposal to expand the existing urban grids. The Urban-Organization decides on a population center in the same fashion that the Rural-Organization does. Because the Urban-Organization decides on a percentage of the population in that center that it wants to expand the grid to as it is not realistic to create the whole grid at once for such a large population. The Agent then calculates the proposal cost as the capital cost needed to expand the grid plus the net present value of the annual cost of operation, maintenance and fuel of supplying the demand of the population desired over a project life of 20 years.

The Government receives one proposal from each organization each time period of the model. In our initial model two approaches are offered for the Government to make its decision. The first and most simple approach is cost. Here the Government simply looks at the lowest net present cost of each proposal and decides to fund it. The second approach takes into consideration the amount of people served by the expenditure with the Government funding the lowest net present cost per capita. The organization that gets the funding proceeds with building the project. The time in the model steps once, repeating the whole process again until $100 \%$ of the population is served.

Many different decision-making parameters can be used here. The user of the model can quickly and easily code a different decision path by using the variables already available in the model. Some possibilities of paths users may want to explore are: the quickest path to achieving universal electrification, the lowest emissions paths to achieving electrification, a weighed combination of cost, number of people served per time step 


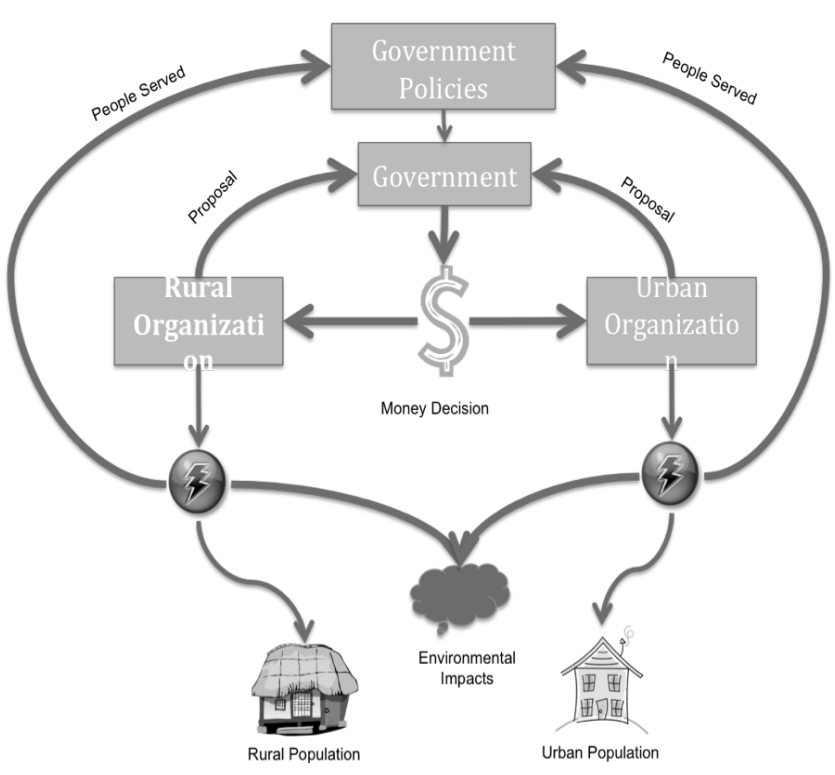

Figure 1: Hierarchical view of the model

and environmental concerns. Interview with policy makers can strengthen the choice of parameters and the weights assigned to them.

A hierarchical view of the model can be seen in Figure 1 . This figure shows the way information and decisions travel through agents and the world.

\section{OutPuT}

NetLogo provides several possibilities to report the model operation to the user through the GUI. The main output of the model is the map of the world that depicts the agents and their locations. The model also reports the percentage of the total population that is being served and segregates the population with electricity between rural and urban for the modeler to observe the demographics of the electrification process. A screen capture of our proof of concept model can be seen in Figure 2.

As stated before the strength of this model is its flexibility and ease of creating different scenarios quickly to analyze the impact on the general trends. As the model gets refined these scenarios can be shared with stakeholders easily and quickly in an intuitive way. The output of the model allows the scenarios to come to life in graphic representations of the world.

The model created can provide results quickly. Users can analyze costs, decision process, population size, and number of cities and percent of population in urban areas in minutes and make general comparisons. The user can develop any number of visualization tools in the main GUI to show those results.

\section{APPLICATIONS}

The interesting part of utilizing the model is to change the different parameters to affect changes in the general observations. By doing that, policy that can help rural populations gain traction, long term costs be reduced, and environmental impacts minimized can start to take shape in an interactive and graphical way. Further, changing the generalized world to reflect actual countries also provides interesting results that need to be explored on a case-by-case basis. We now look at some of these applications of the model.

\section{A. Parameter changes}

Many parameters in the model are easily changed from the main GUI. The user can start by changing the number of population centers as well as the total population. Users should be aware that because the population is being distributed by random numbers following a normal distribution, the actual population in the world might be slightly different than the specified.

Further scenarios can be created by changing the capital and maintenance costs of each renewable energy resource. The default values for the different costs come from the EPA database (EPA, 2010). Diesel cost was set at a default of $\$ 5$ per gallon arbitrarily from observations in Western Africa. Further changes could include the total amount of money available to the government and the number of organizations competing for that money. In developing countries, nongovernment organizations (NGO) can take important roles in the provision of services to the population. Allowing the Population-Centers to make decisions about what source of energy they prefer can provide a more realistic model. Changing not just the cost parameters but who bears the cost of the projects can be a significant source of insight. Finally, in developing countries, especially in rural areas, the price of fuel does not dominate demand but the income level of the population does (Bhattacharyya \& Timilsina, 2010). This is yet another source for parameter changes that can be explored.

\section{B. GIS extension}

A more interesting application is the use of the GIS extension available in NetLogo. This extension is simple to use and a general code to utilize our model with the extension has already been created. The benefits of this extension are the improvement of the general data provided for the model to analyze as well as a realistic visual representation of the data.

The extension can load the data from shape and vector files and draw a world that follows the coordinates in the projection defined by the user. The properties of the shape files can be applied to Agents in the model. So, in our case the world would be drawn to represent the country or region in the GIS data. Population centers would be assigned to the coordinates desired and the population numbers can be assigned to those centers by using census data in the table of properties of the GIS files.

To provide an example, the data for the country of Liberia in West Africa has been used to make a specific scenario of our model. A screen capture of the models map can be seen in Figure 3. In this application of the model the population centers reside in the centroid of the political divisions of the country of Liberia. These centers have the data of the Liberian census of 2008 (Republic of Liberia, 2008). Only one Urban-Center exists since the capital of Liberia, Monrovia, is the only urban district in the country.

Flexibility to input more detailed data is available. For example, instead of using general average values for the 


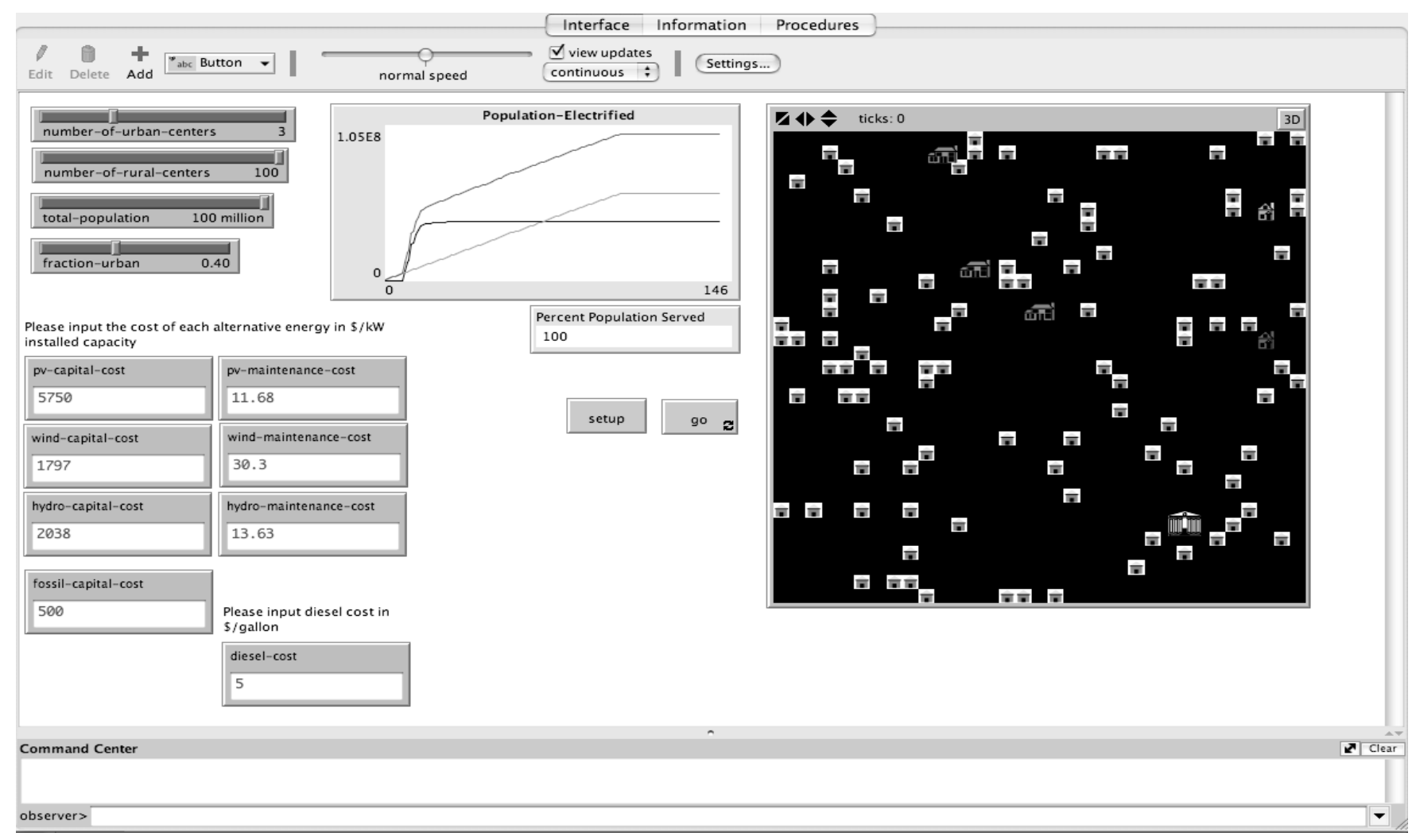

Figure 2: Screen capture of electric grid planning model

countries parameters and IEA figures for demand the data can be segregated to each political division and assign the agents actual data obtained from the census or from interviews. Also, instead of allowing each population center to have a microgrid the agents can be given the choice to propose their own grid or connect to larger populations near by. The same can be done in Monrovia by allowing the fossil fuel based grid to connect to the outlying populations for energy import and export between the population centers. Finally, GPS coordinates of actual power projects can be provided and coded as agents that can interact in the world.

\section{VALIDATION}

Validating the model is a key part of our work. The quality of a model will determine the ability of policymakers to craft appropriate policies for the desired outcomes (Urban et al., 2007). A quality model does not guarantee good policy but a bad model does guarantee bad policy. However, our work is different to general modeling efforts in that we do not seek a forecast of the future.

With this model we seek to provide stakeholders with a tool for scenario building that holds the best information we can make available to them. In this way policy makers can have productive assessments of their ideas and are able to see the outcomes of the scenarios they propose.

Because of this, our validation efforts focus on the quality of the data present in the model and on the involvement of stakeholders in the modeling process. A strong tool for validation of our model is to convert the $\mathrm{ABM}$ into a roleplaying model. By doing this the "black box" that ABM seems to be can become quite clear to the stakeholders and other researchers (Barreteau, Bousquet, \& Attonaty, 2001).

Because decision-making varies widely from country to country, there is a need to validate the model in each application of it to a different region. To apply and validate our model, we propose two phases. The first is to conduct research with the policy makers and officials involved in a particular country. This initial research sheds light on the decision processes that needs to be coded in the model and the actual decision-making parameters that will inform those processes.

Once these processes are clear, the second phase involves allowing the stakeholders to take control of an agent. In this exercise the parameters, information and decisions of each agent are fed to a GUI. This way the users can play the game by actually making the decisions for the agent informed with the data in the model. This process is a powerful validation tool since the main uncertainty of decision-making by humans is now reduced.

Allowing humans to play the game helps the coder identify any issues with the model and any data that might be missing for the agents to make informed decisions. It also gives accountability to the stakeholders since the way their decisions are carried out in the model are no longer hard-coded into the model. The game playing validation is then dependant on the honesty of the stakeholders that will use it later.

Netlogo provides an extension that allows this conversion easily: HubNet (Wilensky \& Stroup, 1999). HubNet can also go beyond being a validation tool but actually become the main use for the model. Using HubNet policy analysis 


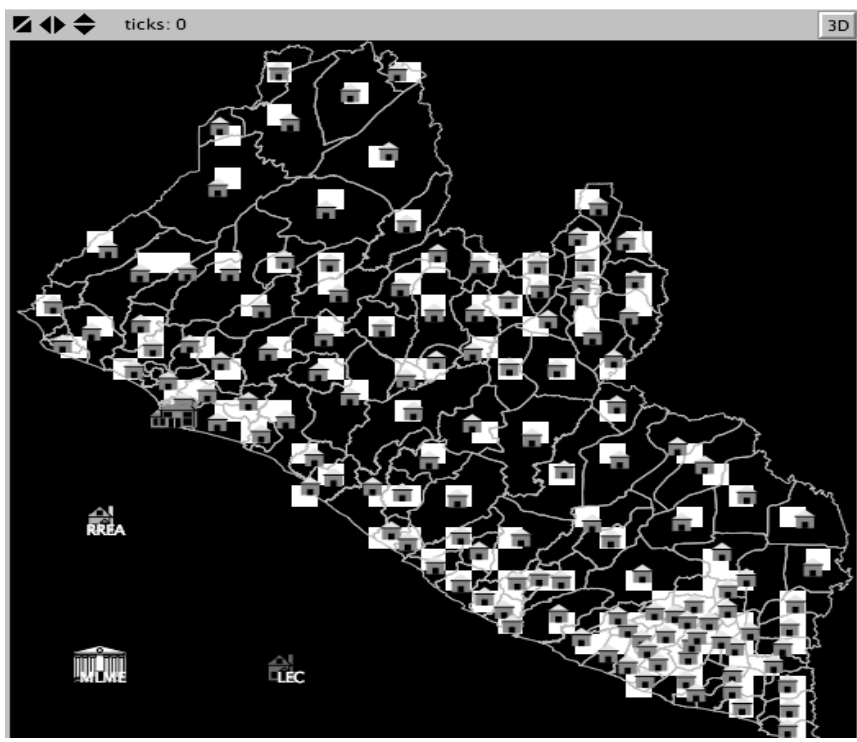

Figure 3: GIS enhanced model implementation, Liberia, West Africa

sessions can be conducted that allow stakeholders to see the possible outcomes associated with the policies being crafted. Our validation efforts will be carried out following these two phases once a more robust model is in place.

\section{REFINING THE MODEL}

The model's deficiencies are primarily in the decision making process and the time flow in the model. The decision making process in the model is simple, something useful for understanding the general work of the agents. However, a more complex decision process should be used. Additional metrics should be added to the Government's decision as well as the Organization's. The organizations presently do not learn from their previous paths. They should be allowed to learn, especially when they loose a bid, to attempt a better proposal during the subsequent time period. Finally, the Government should be trying to optimize its decision based on certain metrics. This should be coded into the model but will be different when users start building applications for individual countries or regions and should therefore be reviewed on a case-by-case basis.

Presently the time in the models advances once for every loop of decisions that is made. The projects are also all completed in one time period. An improvement to this mechanism will be sought since the time in which proposals are crafted, decisions are made and energy projects are implemented are different. Further, the organizations may receive funding for more than one proposal at a time but their building efforts will be limited by the size of their manpower to actually implement the build.

\section{CONCLUSIONS}

We conclude that ABM coupled with GIS is a promising approach to create a tool that creates scenarios for policy makers and stakeholders when planning the electrical infrastructure in developing countries. We have also found that the flexibility of $\mathrm{ABM}$ allows the incorporation of developing countries unique characteristics into the model and easy adaptation for implementation of the model in different countries. Finally, validation of ABM models for this purpose depends on the involvement of stakeholders. Far from being a limitation, this is a strength of the modeling process since it introduces accountability the stakeholders must shoulder for the success of the process. It also means that modelers cannot just present a "black box" but must introduce the mechanism of the model with clarity to the stakeholders. This exchange will build trust and help the results become an effective policy-planning tool.

\section{ACKNOWLEDGMENT}

We would like to thank the Center for Sustainable Systems and the Center for the Study of Complex Systems at the University of Michigan for their support to our project.

\section{REFERENCES}

Barreteau, O., Bousquet, F., \& Attonaty, J.-M. (2001). Role-playing games for opening the black box of multi-agent systems : method and lessons of its application to Senegal River Valley irrigated systems. Journal of Artificial Societies and Social Simulation, 4(2).

Bhattacharyya, S. C., \& Timilsina, G. R. (2010). Modelling energy demand of developing countries: Are the specific features adequately captured? Energy Policy, 38(4), 1979-1990. Elsevier. doi: 10.1016/j.enpol.2009.11.079.

EPA. (2010). Renewable Energy Cost Database.

Garcia, R. (2005). Uses of Agent-Based Modeling in Innovation/New Product Development Research. Journal of Product Innovation Management, 22(5), 380-398. doi: 10.1111/j.1540-5885.2005.00136.x.

International Energy Agency. (2010). Energy Poverty: How to Make Modern Energy Access Universal? Outlook. Paris.

Reiche, K., Covarrubias, A., \& Martinot, E. (2000). Expanding electricity access to remote areas: off-grid rural electrification in developing countries. World Power. Retrieved February 1, 2011, from http://users.tkk.fi/ apoudyal/Session 20 Reading Reiche_et_al_WP2000.pdf.

Republic of Liberia. (2008). 2008 Population and Housing Census. Monrovia, Liberia.

Urban, F., Benders, R. M. J., \& Moll, H. C. (2007). Modelling energy systems for developing countries. Energy Policy, 35(6), 3473-3482. doi: 10.1016/j.enpol.2006.12.025.

Wilensky, U. (1999). NetLogo. Evanston, IL: Center for Connected Learning and Computer-Based Modeling, Northwestern University. Retrieved from http://ccl.northwestern.edu/netlogo/.

Wilensky, U., \& Stroup, W. (1999). HubNet. Evanston, IL: Center for Connected Learning and Computer-Based Modeling, Northwestern University. Retrieved from http://ccl.northwestern.edu/netlogo/hubnet.html.

Zerriffi, H., \& Wilson, E. (2010). Leapfrogging over development? Promoting rural renewables for climate change mitigation. Energy Policy, 38(4), 1689-1700. Elsevier. doi: 10.1016/j.enpol.2009.11.026. 